\title{
Big data to support Indonesian health system recovery from the pandemic: potential and challenges
}

\author{
Anis Fuad $^{1 *}$ \\ ${ }^{1}$ Department of Biostatistics, Epidemiology and Population Health, Faculty of Medicine, Public Health and Nursing, Universitas Gadjah \\ Mada, Yogyakarta, Indonesia
}

\begin{abstract}
The Covid-19 pandemic has had a tremendous impact on the Indonesian health system. At the peak of the second wave, health care crises occurred in various regions, causing difficulties in accessing essential and emergency health services, accompanied by increased deaths in hospitals and self-isolation. Various efforts have been made to overcome the crisis, from providing field hospitals, adding facilities and infrastructure in hospitals, empowering health workers and volunteers, logistics, and implementing a massive digital system. In addition, strengthening in the upstream aspects starting from tracing, testing to health protocols is also continuously encouraged, along with the acceleration and expansion of vaccinations. The pandemic has encouraged massive utilization of digital systems in various aspects accompanied by an extraordinary increase in the volume of digital data in various types and quality. The critical question then is, what are the opportunities for big data to support the recovery of the health system affected by the pandemic? Likewise, what challenges must be overcome to make optimal use of big data so that the Indonesian health system can quickly recover, grow and become resilient again? To answer the questions above, we reviewed bibliographic databases, gray literature, media, and webinar recordings available online. Next, we group their potential based on the building blocks of the health system. Using the e-health framework from WHO-ITU, we categorize the main challenges of using big data into seven major groups: governance, strategy, data standards, interoperability, application, regulation, and human resources. We provide special notes on each aspect of these challenges along with priority follow-up steps. In the end, the Covid-19 pandemic provides essential lessons for the Indonesian health system to take advantage of digitalization, especially big data intelligently and creatively, to encourage the immediate recovery of the health system. However, several significant challenges need to be overcome so that big data can be utilized optimally to overcome this big global problem.
\end{abstract}

\footnotetext{
*Corresponding author: anisfuad@ugm.ac.id
} 